\title{
RADIATIVE PION CAPTURE IN NUCLEI *
}

\section{NOTICE}

This report was prepared as an account of work This report was prepared States Government, Neither sponsored by the United States Sover Atomic Energy the United States nor the United States Aromic Ener Commission, nor any of their employees, nor any of their contractors, subcontractors, of their employees, makes any warranty, express or implied, or assumes any legl tiability or responsibility for the accuracy, comlega iss pieteness or usefus disclosed, or represents that its use product or process divelosed, or represents
Helmut W. Baer and Kenneth M. Crowe

Lawrence Berkeley Laboratory

University of California

Berkeley, California 94720

The process of radiative pion capture has in recent years been shown to be a good probe of nuclear structure. The experiments and theoretical results which support this statement are reviewed. High-resolution data on the photon spectra from nuclei ranging in mass $1 \leq A \leq 209$ are shown. We discuss the observation of giant resonances in $\mathbf{T}_{\mathbf{z}}=+1$ nuclei, the quasi-free capture process, and transitions to individual bound nuclear states. The observed transition rates in 1p-shell nuclei are compared with recent shell model calculations based on an impulse-approximation Hamiltonian whose amplitudes are not adjustable constants, but are obtained from the fundamental process on the nucleon, $\pi^{-}+p \rightarrow n+\gamma$. The agreement is good within the limits set by the uncertainties of pionic $x$-ray data. Some features of radiative $\pi$ capture on very light nuclei $-A=2,3$ and 4-are reviewed. Finally, we discuss the $\pi^{-}+{ }^{6} \mathrm{Li}\left(1^{+}\right) \rightarrow \gamma+{ }^{6} \mathrm{He}\left(0^{+}\right)$transition where the PCAC hypothesis and soft-pion limit have been applied. The $1 \mathrm{~s}-\mathrm{state}$ radiative capture rate evaluated with these assumptions is found to agree with impulise approximation calculations and is within reasonable agreement with the recent data when corrections for $2 p$ capture are made.

\section{INTRODUCTION}

The question to which we draw your attention is: what can be learned about nuclear structure by stopping negatively charged pions in a target and examining with $2-\mathrm{MeV}$ resolution the emitted photon spectrum in the region of 50 to $140 \mathrm{MeV}$ ? Processes which produce photons in this region are radiative capture:

$$
\begin{aligned}
\pi^{-}+A_{i} & \rightarrow A_{f}+\gamma \\
& \rightarrow(A-1)_{f}+n+\gamma \\
& \rightarrow(A-2)_{f}+n+n+\gamma \\
&
\end{aligned}
$$

and charge exchange

$$
\pi^{-}+A_{i} \rightarrow A_{f}+\pi^{0} 2_{y}
$$

The latter has only been observed with hydrogen and $3 \mathrm{He}$. Study of hydrogen and deuterium in the early 1950's helped determine basic properties of the pion, such as its mass, odd intrinsic parity, and $\pi N$ coupling strength. In recent years targets ranging up to $A=209$ have been studied and some of the interest has turned to the study of nuclear structure.

T'he study in 1966 by Davies et al. ${ }^{1}$ with a large NaI detector demonstrated that the branching ratios for reactions (1) for pions in bound atomic orbits is $\sim 2 \%$. A summary of measured values for both reactions (1) and (2) is given in Table $I$. The basic fact established by observation of the high-energy photons is that the reactions of Eq. (1) occur as a direct interaction (rather than through formation of a compound nuclear state) of a $\pi^{-}$with a proton in the nucleus, the fundamental process being $\pi^{-}+p \rightarrow n+\gamma$. With this knowledge one could anticipate that the impulse approximation might be valid, and thus one had a :easonably good idea of what transition operator to use in calculating the radiative transition for nuclei. Recognizing the similarity between the dominant term of this operator,

$\tau_{+} \vec{\sigma} e^{i \vec{k} \cdot \vec{r}}$, with the electric dipole operator in photonuclear reactions, several author $\mathrm{s}^{2}$ predicted that the well-known giant-dipole resonances should be selectively and strongly excited. Motivated by these predictions, the Berkeley group 3 set up a high-resolution electron-posi-

\section{* Work performed under the auspices of the U. S. Atomic Energy Commiseion.}


Table I. Radiative and charge exchange branching ratios ${ }^{\mathrm{n}}$ for stopped $\pi^{-}$abxorption on nutci.

\begin{tabular}{|c|c|c|c|}
\hline Target nuclcus & $\begin{array}{l}\text { Radiative }\left(\mathbf{R}_{\gamma}\right) \\
\{\%)\end{array}$ & $\begin{array}{c}\text { Charge exchange }\left(\mathrm{R}_{\pi} \mathrm{O}\right) \\
(\mathrm{N})\end{array}$ & $\begin{array}{l}\text { Alonic captureb } \\
\text { crlitals }\end{array}$ \\
\hline${ }^{\prime} \mathbf{H}$ & $39.5 \pm .3^{\circ}$ & $60.5 \pm .3^{\circ}$ & Is \\
\hline $2 H$ & $24.7 \pm .7^{c}$ & $.08 \pm .10^{d}$ & Is \\
\hline${ }^{3} \mathrm{He}$ & $13.9 \pm 1.9$ & $18.6 \pm 2.3$ & $1 \mathrm{~s}, 2 \mathrm{p}$ \\
\hline${ }^{4} \mathrm{He}$ & $1.50 \pm .09$ & Q forbidden & Is. $2 \mathrm{p}$ \\
\hline${ }^{5} \mathrm{I} . \mathrm{j}$ & $4.39 \pm .58$ & $<+\infty{ }^{\circ}$ & 13. 2p \\
\hline${ }^{7} \mathrm{Li}$ & $1.9 \pm .2^{f}$ & $.003^{c}$ & $1 s, 2 p$ \\
\hline $12 \mathrm{C}$ & $1.92 \pm .2 n$ & Q forbidden & 15. $2 p$ \\
\hline${ }^{14} \mathrm{~N}$ & $2.24 \pm .48$ & $<.5 \%$ & $1 \mathrm{~s}, 2 \mathrm{p}$ \\
\hline${ }^{16} \mathrm{O}$ & 2.244 .48 & Q forbidden & Is. 2p \\
\hline${ }^{24} \mathrm{Mg}$ & $2.15 \pm .43$ & $Q$ forbidden & $15.2 p(3 d)$ \\
\hline${ }^{32} \mathrm{~S}$ & $1.8 \pm .1^{f}$ & & 2p. 3d \\
\hline${ }^{10} \mathrm{C}_{2}$ & $1.94 \pm .35$ & $<.5 \%$ & $2 p \cdot 3 d$ \\
\hline${ }^{63,65} \mathrm{Cu}$ & $1.5 \pm .1^{f}$ & & $2 p .3 d$ \\
\hline${ }^{209} \mathrm{Bi}$ & $1.57 \pm .52$ & $<54$ & $+f, 5 p$ \\
\hline
\end{tabular}
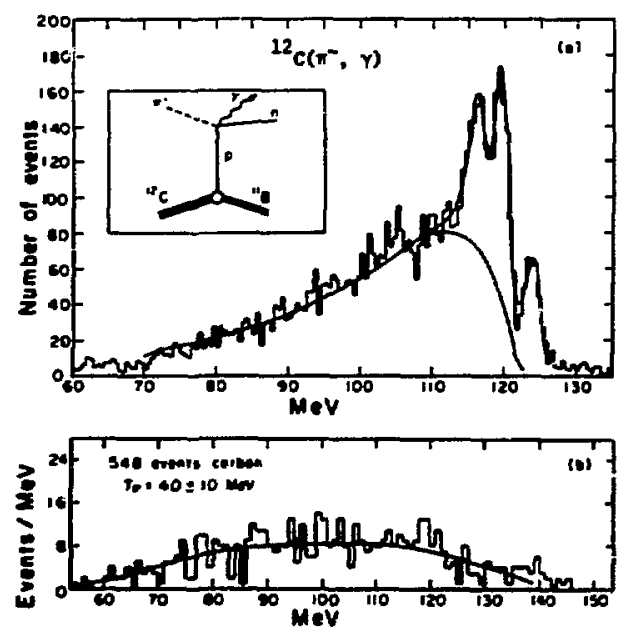

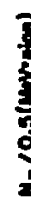

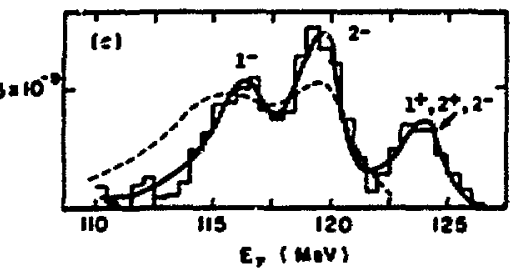

$x$ 733-307 tron pair spectrometer at the LBL 134 inch cyclotron, and were able to observe the predicted sharp lines in the ${ }^{2} \mathrm{C}\left(\pi^{-}, y\right)^{12} \mathrm{~B}$ reaction (Fig. 1$)$.

In the four years since then, the field has developed rapidly. Experimentally, the data have been extended to nuclei of the entire periodic table and several tranaition. to individual nuclear states isolated. The pionic $x$-ray data, needed to determine the capture orbital. and total strong absorption widthe, have improved. Theoretically, the relative im portance of various terms in the effective interaction has been further clarified and more realistic nuclear wave functions are being employed. These efforts, as will be seen, have shown radiative $\pi$ capture to be a quantitative probe of nuclear structure.

Fig. 1. Photon energy spectrum from $\pi$ - capture in $12 \mathrm{C}$. (a) Spectrum with fitted function, using three Breft-Wigner forms plus the pole model for the continuum. (b) Photon spoctrum for pions with a mosen energy of $10 \mathrm{MeV}$ used for inflight beckground suberaction. (c) Spec trum with the pole model subtracted. The solid cur'se is the beat fit; the dashed curve if the prediction al Kelly and Uberall 27 for excitation of giant dipole states. 
In addition to nuclear structure investigations, the experimental $\pi^{-}$capture matrix elements contribute to developing the theory which in the literature is termed the elementary particle treatment ${ }^{5}$ of nuclei. Here onc tries to link together various weak and electromagnetic proces ses such as radiative and charge-exchange T capture, $\mu$ capture, $\beta$ decay, $\gamma$ decay, and electron scattering. The nuclear physics is contained in various invariant form factors of the vector and axial-vector weak hadronic currents. By making use of the conserved vector current (CVC) and partially conserved axial vector-current (PCAC) hypotheses, together with the soft pion limit $(q \rightarrow 0)$, one can derive relationship between these rates. For example, it has been shown that radiative T capture from the 1 s orbital is governed by the axial-vector form factor, which also appears (at a lower momentum trans fer) in allowed Gamcw-Teller $\beta$-transi tione and in $\mu$ capture. This type of study was initiated in 1965 by $\mathrm{Kim}$ and Primiakoff 6 and has been advocated recently by numerous authore. 5 At present only two transitions, in ${ }^{3} \mathrm{He}$ and ${ }^{\mathrm{Li}}$, have been carefully studied in this manner and these will be discussed. Two facts limit the greater application of this approach. In light nuclei, $2 \mathrm{p}$-state capture dominates and for this orbit the soft-pion results do not obtain. Also, the need to have as input good experimental rates on the two atates (or anslogs) connected by $\pi$ capture has restricted the application of this approach to a few cases.

\section{EXPERIMENT}

The process of radiative pion capture and the method of observation to be dis cussed occur in the laboratory in the fol lowing manner. A beam of $100-\mathrm{MeV}$ pione, produced by bombarding a Be ribbon with $720-\mathrm{MeV}$ protons, is brought to a focus at a point $10 \mathrm{~m}$ frum production. The transit time is $4.1 \times 10^{-8} \mathrm{sec}$, the pion lifetime is $y \tau_{1} / 2=4.5 \times 10^{-8}$ sec. Before entering the target, the pions are dezraded in energy by $\mathbf{a}-25-\mathrm{cm}$ length of $\mathrm{CH}_{2}$ and brought to rest in a target of
$1-4 \mathrm{~g} / \mathrm{cm}^{2}$. The slow-dowr, time is $-2 \times 10-9$ sec. When the pion kinetic ene rgy has dropped into the $\mathrm{gV}$ region it makes a transition to a negative energy atomic orbit of the nuclcus to be $n$ tudied. There it quickly $\left(10^{-13}\right.$ to $\left.10^{-11} \mathrm{sec}\right)$ makes 50 to 100 Auger or $x$-ray tranai tione until it reaches either the $1 \mathrm{~s}$ state or a higher orbit which has sufficient overlap with the nucleus to make absorption corspetitive with $x-r a y$ transition. About $2 \%$ of the pions ahsc,rbed produce photons with energies centered around $120 \mathrm{MeV}\left(m_{\pi}=139.6 \mathrm{MeV}\right)$. The energy spectrum by ricasured by permitting the photons to impinge on a thin gold foil, part of a pair speitrometer, where $2.3 \%$ produce electron-position pairs. The circular trajectories of this pair in an - 8-kG field are measured at several pointe with wire sparik chambers. The momenta thus measured are added to give the photon energy. The pair spectromete $r^{4}$ employed in this measuremente at the 184 -inch Ber'seley cyclotron gives a resolution (F (WHM) of $2 \mathrm{MeV}$ at $130 \mathrm{MeV}$. The overall efficiency (conversion $x$ $\Delta \Omega / 4 \pi)$ is $4.1 .5 \times 10^{-5}$ at $130 \mathrm{MeV}$.

\section{GENEIRAL ASPECTS OF THE PROBLEM}

For orientation, let v.s look at some aspects of the physics isvolved which pertain to all nuclei and which are already evident from the ${ }^{12} \mathrm{C}$ study.

\section{TREATMENT OF THE CONTIRUUM}

All epectra for nuclei with $A>4$ and $A=3$ exhibit a broad continuum with $a$ maximum between 110 and $120 \mathrm{MeV}$. Compared to three-body phase space (Afny) the data are more sharply peaked (Fig. 5a); compared to a Fermi-gas model calculation ${ }^{1,4}$ the data are broader. A reasonably good description is given by the pole model of Dakhno and Prokoshkin 7 which identifies the continuum with quasifree capture on 2 proton. The single-pole diagram (discussed by Shapiro ${ }^{7}$ in the context of dispersion theory for direct nuclear interactions) and the expression for the photon spectrum are: 
amplitude $=\frac{G_{g}}{\left(t-m^{2}\right)}$,

4-momentum transfer: $t=\left(P_{y}+P_{n}-P_{\pi}\right)^{2}$,

proton (neutron) [ pion] $\operatorname{mass}=m\left(m_{n}\right)[\mu]$

proton separation parameter:

$$
\Delta=M+m_{n}+E_{A-1}^{*}-M_{A} \text {. }
$$

$E^{\prime}=$ total energy.

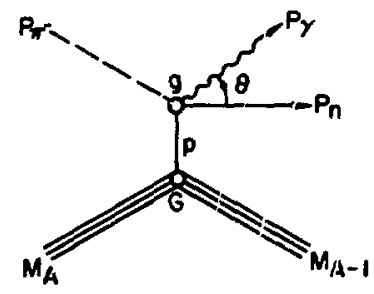

XeL $734-2000$

Fig. 2. Pole graph for raciative $\pi^{-}$ capture.

$\frac{d N\left(E_{Y}\right)}{d E_{Y}}=\operatorname{const} . \times(G \cdot g)^{2} \times E_{Y}^{2} \int_{-1}^{+1} d(\cos \theta) \frac{E_{n} P_{n}^{3}}{\left(t-m^{2}\right)^{2}} \times \frac{E_{A-1}}{P_{n}^{2}\left(M_{A}+\mu-E_{Y}\right)+P_{n} E_{Y} E_{n} \cos \theta}$

The curves shown with the data were computed with the above expression, which we evaluate numerically to include the complete kinematica. Since the normalization and the average excitation ene rgy Et ${ }_{-1}$ of the recoil nucleus have not been given by theory, we choose values which give the best description of the data. The values of $E_{A-1}^{*}$ determined in this manner are found to v : $y$ between 0 and $5 \mathrm{MeV}$. The pole model is clearly a fir at approximation: it ignores the motion of the proton in the nucleus and the final state interaction between the outgoing neutron and recoil nucleus. These are known to modify considerably the photon spectrum of ${ }^{2} \mathrm{H}$ and ${ }^{2} \mathrm{He}$ as discussed below. Neverthelees, one obtains a good description of the photon spectrum in many cases with particularly good results for ${ }^{3} \mathrm{He}$ and ${ }^{1} \mathrm{~N}$. Ultimately one hopes for a unified model which treats both quasi-free capture and the resonances on an equal footing.

\section{THE EFFECTIVE HAMILTONIAN}

The tranaition operator for radiative $\pi$ capture is taken to be the nonrelativistic reduction of the photopion production amplitude on the nucleon (Chew et al. ${ }^{40}$ ). In applying this to nuclei in the impulse approximation (IA), and taking the pion to be bound in an atomic orbit $\phi ?(\vec{r})$. Delorme and Ericson ${ }^{2}$ write this as

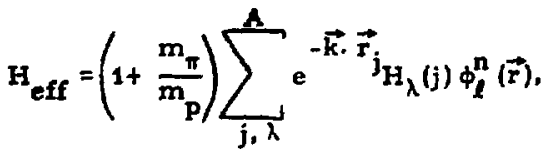

where

$$
\begin{aligned}
& H(j)=2 \pi i^{+}(j)\left[A \vec{\sigma}_{j} \cdot \varepsilon_{\lambda}+B\left(\vec{\sigma}_{j} \cdot \varepsilon_{\lambda}\right)(\vec{q} \cdot \vec{k})\right. \\
& \left.+C\left(\vec{\sigma}_{j} \cdot \vec{k}\right)\left(\vec{q} \cdot \varepsilon_{\lambda}\right)+i D \vec{q} \cdot \overrightarrow{k i k} \times \varepsilon_{\lambda}\right) \\
& +E\left(\vec{\sigma}_{j} \cdot \vec{q}\right)\left(\vec{q} \cdot \varepsilon_{\lambda} \| \sigma\left(\vec{r}-\vec{r}_{j}\right) .\right.
\end{aligned}
$$

The transition rate (in units of $n=c=1$ ) be tween initial $\left(J_{i}\right)$ and final $\left(J_{f}\right)$ nuclear states is given by

$\Lambda_{y}(n l ; i \rightarrow f)=\frac{k}{\pi} \frac{1}{\left(2 J_{i}+1\right)(2 l+1)}$

$\times \sum_{m^{\prime} a} \int \frac{d R}{4 \pi}\left|\left\langle J_{f} M_{f}\left|H_{e f f}\right| J_{i} M_{i}\right\rangle\right|^{2}$

In the above, $\vec{\sigma}$ and $\vec{r}_{\text {f }}$ are the Pauli spin and coordinate of the $j$ th nucleon and $t^{+}(j)$ is the isospin raising operator; i. e. . $t^{+} \mid$proton $\rangle=\mid$neutron $\rangle$. The photon polarization and propagation vectore are denoted by $\vec{\varepsilon}_{\lambda}(\lambda= \pm 1)$, and $\vec{k}$ and the pion momentum and coordinates are denoted by $\vec{q}+$ and $r$. The effective coupling con-

tIn practice one takes $\vec{q} \phi_{l}^{n}=-i \vec{\nabla} \phi_{l}^{n}$. The Fermi motion of the proton on which capture takes place ia ignored. A discussion of this point can be found in Ref.9. 
stants $A, B, C, D$, and $E$ are linear combinations of the electric and magnetic multipoles contributing to the $\gamma+n \rightarrow \pi^{-}+p$ cross section at low energies. Threshold values have been given by nume rous authors and some are listed in Table II. Although the most recent solutions $(1972-73)$ are all based on the tables of Berends et al. 12 (1967), there are still some discrepancies in $B, C, D$, and $E$. Kawaguchi et al. 8 determined their values by employing the CGLN 40 solution of the fixed-t dispersion relations and used as input pion-nucleon phase shifts. The fact that these values agree quite closely with the ones based on the work of Berends et al. , which reproduce photo production data, gives a rather consistent picture. The need for a sign change in the $D$ term has just recently been suggested by Maguire and Werntz 9 and is corroborated by Nixon. 10 Also, the kinematic factor $1+m_{\pi} / m_{0}$ which appears in Eq. (4) but not in the work of Delorme and Ericson and Kawaguchi et al. is discussed in Ref. 9.

Most of the calculations for $\pi$-capture transitions have employed only the $A\left(\vec{\sigma} \cdot \hat{E}_{\lambda}\right) e^{i R} \cdot \vec{r}$ terms of the Hamiltonian, since an estimate by Delorme and Ericson ${ }^{2}$ indicated that the momentumdependent terms play only a minor role for $1 \mathrm{~s}, 2 \mathrm{p}$, and $3 \mathrm{~d}$ capture. This result was derived for the total (all final nuclear states) radiative capture rate by employing the Fermi gas model and closure over final nuclear states. Recent atudies $13,14,9$ in which transitions to individual nuclear states were considered have shown that this conclusion is correct to within a few percent for 1a-state capture; however, for $2 p$-state capture the momentum-dependent terms (B, C,D) make sizeable contributions. For example, in the $\pi^{-}+{ }^{6} \mathrm{Li} \rightarrow \mathrm{y}+{ }^{6} \mathrm{He}$ (g.s.) transition Vergados and Baer ${ }^{14}$ obtain a value $f=0.7$ for the ratio of contributions to $\lambda_{y}(2 p)$ involving $B$ and $C$ terms to the $A$ term only. The $E$ term, quadratic in pion momentum, is expected 14 to make negligible contributions to $1 \mathrm{~s}$ and $2 \mathrm{p}$ capture, but may be important for $3 d(\ell \geq 2)$ capture.

\section{ROLE OF PIONIC X-RAY DATA}

The physics of mesic atoms and the pionic $x$-ray data play an absolutely crucial role in the quantitative evaluation of theoretical $\pi$-capture matrix elements. Two quantities are needed: (1) probabilities, $\omega(n)$, that the pion gets absorbed into the nucleus from the orbit ( $n l$ ) and (2) total strong absorption rates $\Lambda_{a}(n)$ ) of each orbit. The $\omega(n)$ are relilted to the level population probabilities $P(n)$ by

$$
\omega(n l)=P(n l) \frac{\Lambda_{\mathrm{a}}(\mathrm{nl})}{\Lambda_{\mathrm{a}}(\mathrm{nl})+\Lambda_{\mathrm{x}}(\mathrm{nl})+\Lambda_{\mathrm{A}}(\mathrm{nl})},
$$

where $\Lambda_{x}(n l)\left[\Lambda_{A}(n l)\right]$ are the $x-r$ ay (Auger) transition rates for depopulating level $n l$. The $P(n l)$ are determined by atomic cascade calculations which fit to the $x$-ray intensity data. The $\Lambda_{a}(n)$ are either measured directly from the broadening of $x-r$ ay lines or deduced indirectly from the $x$-ray intensity balance on level ( $n$ l) and the electromagnetic width. The fraction of pions undergoing radiative $\pi$-capture transitions at each

Table II. Coupling constants of the effective impulse approximation Hamiltonian (text) for radiative $\pi^{-}$capture transitions in nuciei.

\begin{tabular}{|c|c|c|c|c|c|}
\hline & $\stackrel{A}{\left(10^{-3} \mathrm{~m}_{\pi}^{-2}\right)}$ & $\left(10^{-3} \mathrm{~m}_{\pi}^{-4}\right)$ & $\begin{array}{c}C \\
\left(10^{-3} \mathrm{~m}_{\pi}^{-4}\right)\end{array}$ & $\begin{array}{c}D \\
\left(10^{-3} \mathrm{~m}_{\pi}^{-4}\right)\end{array}$ & $\frac{E}{\left(10^{-3} m_{\pi}^{-4}\right)}$ \\
\hline Delorme E Ericson ${ }^{2}$ (1966) & $.34 \pm 3$ & 19 & .17 & -10 & \\
\hline Kawaguchi of at ${ }^{b}$ (1968) & -32 & 7.5 & -37 & -14 & \\
\hline Maguire \& Wemtze (1972) & -33.2 & 4.8 & -32.9 & +11.7 & 30.4 \\
\hline Nixond (1973) & -33 & 5.5 & -37 & +13 & 30 \\
\hline Roig and Pascualf (1973) & .31 .9 & 4.2 & -29.5 & -10.9 & 20.7 \\
\hline Schwels $(1973)^{e}$ & $-31.7 \pm .9$ & $6.5 \pm .2$ & $-33.0 \pm .9$ & $+8.9 \pm .6$ & \\
\hline
\end{tabular}


orbital is $\omega(n \ell)-\Lambda_{\gamma}(n \hat{x}) / \Lambda_{a}(n \ell)$. Since $\mathrm{n} \ell$ is not fixed in the existing measurements of the radiative branching ratios $\left(R_{y}\right)$ one must perform the incoherent sum over all orbitals

$$
\mathrm{R}_{\gamma}=\sum_{n \ell} \frac{\Lambda_{\gamma^{(n \ell)}}}{\Lambda_{a}^{(n \ell)}} \omega(n \ell) .
$$

It is generally as sumed that the ratio $\Lambda_{y}(n l) / \Lambda_{\mathrm{a}}(n \ell)$ depends only on $\ell$, not $n$. Fdr light nuclei, capture occurs only from $\ell=0$ and $\ell=1$ orbits; therefore, the quantity

$$
R_{\gamma}=\frac{\Lambda_{Y}(1 s)}{\Lambda_{a}(1 s)} \sum_{n} \omega(n s)+\frac{\Lambda_{y}{ }^{(2 p)}}{\Lambda_{a}^{(2 p)}}
$$$$
x \sum_{n} \omega(n p)
$$$$
\text { [light nuclei] }
$$

is compared with experiment. The role of p-state capture in light nuclei is often underestimated. The most recent studies indicate $\mathrm{p} / \mathrm{s}$ state capture ratio

$\left[\sum_{n} \omega(n p) / \sum \omega(\mathbf{n s})\right]$ or $1.2 \pm 0.3$ (Ref.15),

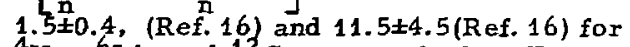
${ }^{4} \mathrm{He},{ }^{6} \mathrm{I}_{\mathrm{i}}$, and ${ }^{12} \mathrm{C}$, respectively. For ${ }^{1} \mathrm{H}$ and ${ }^{2} \mathrm{H}$ it is thought ${ }^{17}$ to be nearly zero.

\section{RESUITS FOR NUCLEI WITH $1 \leq A \leq 4$}

The photon spectra of all light nuclei studied thus far are displayed in Fig. 3 and results are summarized in Table III. [ The only other possibility - an unstable target-is tritium, which the Berkeley group is planning to measure later this year at Los Alamos (LAMPF). ] Since the emphasis here is primarily nuclear structure, we comment only briefly on each spectrum.

${ }^{1}$ H. The spectrum of hydrogen has played a central role in determining the basic properties of the pion and $s-$ wave pion-nucleon interactions. In the present work it serves to calibrate the energy scale, instrumental line shape, and relative and absolute detection efficiency. The Panofsky ratio deduced for the spectrum hown in Fig. 3 a is $1.56 \pm 0.10$, which agrees well with the currently accepted value of $1.53 \pm 0.02$.

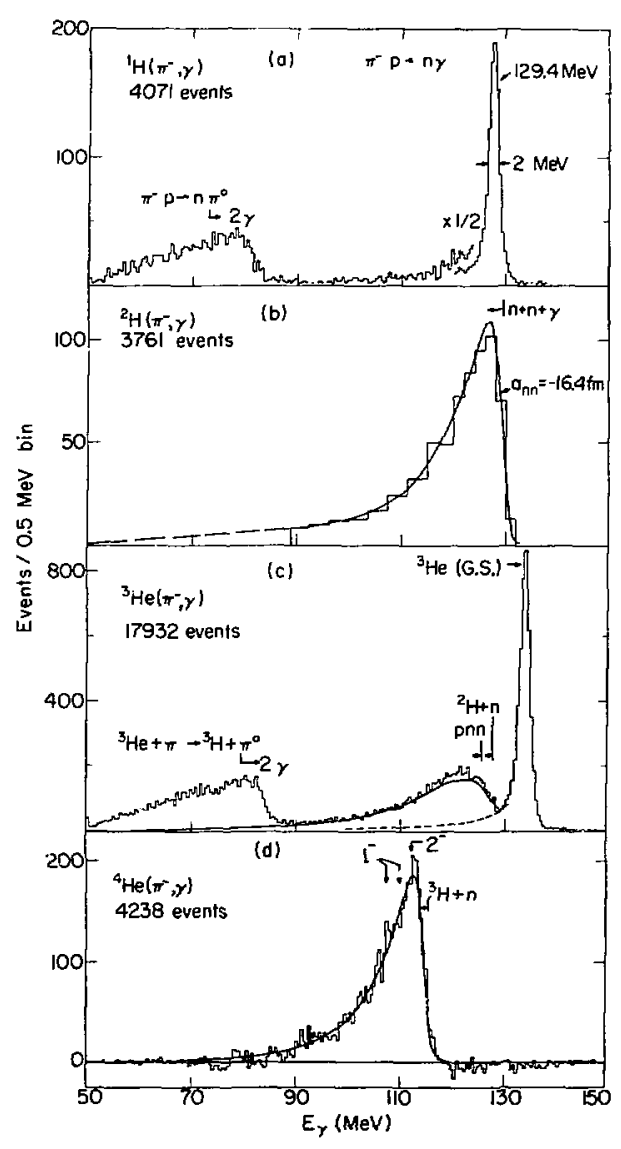

$\times 6 L 733-2430$

Fig. 3. Photon spectra for nuclei with $1 \leqslant A \leqslant 4$. (a) Spectrum for hydrogen (employed in the calibration of the pair spectrometer). (b) Spectrum for denterium from Ryan. 19 The curve is calculated for a 2 -neutron ${ }^{1} S$-state scattering length $a_{\text {mn }}=-16.4 \mathrm{fm}$.

(c) Spectrum for ${ }^{3} \mathrm{He}$. 'the solid curve is a pole-model calculation. (d) Spectrum for ${ }^{4} \mathrm{He}$ by Bistirlich et al. ${ }^{4}$ The solid curve is an R-matrix calculation, assuming excitation of three states as indicated.

${ }^{2}$ H. The spectrum for deuterium was the first to demonstrate the usefulness of radiative $\pi$ capture tc nuclear physics. The spectrum (Fig. 3 b) is sharply peaked toward the high-energy end due to the 
Table III. Summary of experimental values ${ }^{\mathrm{a}}$ and some resulting interpretations for radiative absorption of stopped $\pi^{-}$on nuclei with $1 \leqslant A \leqslant 4$.

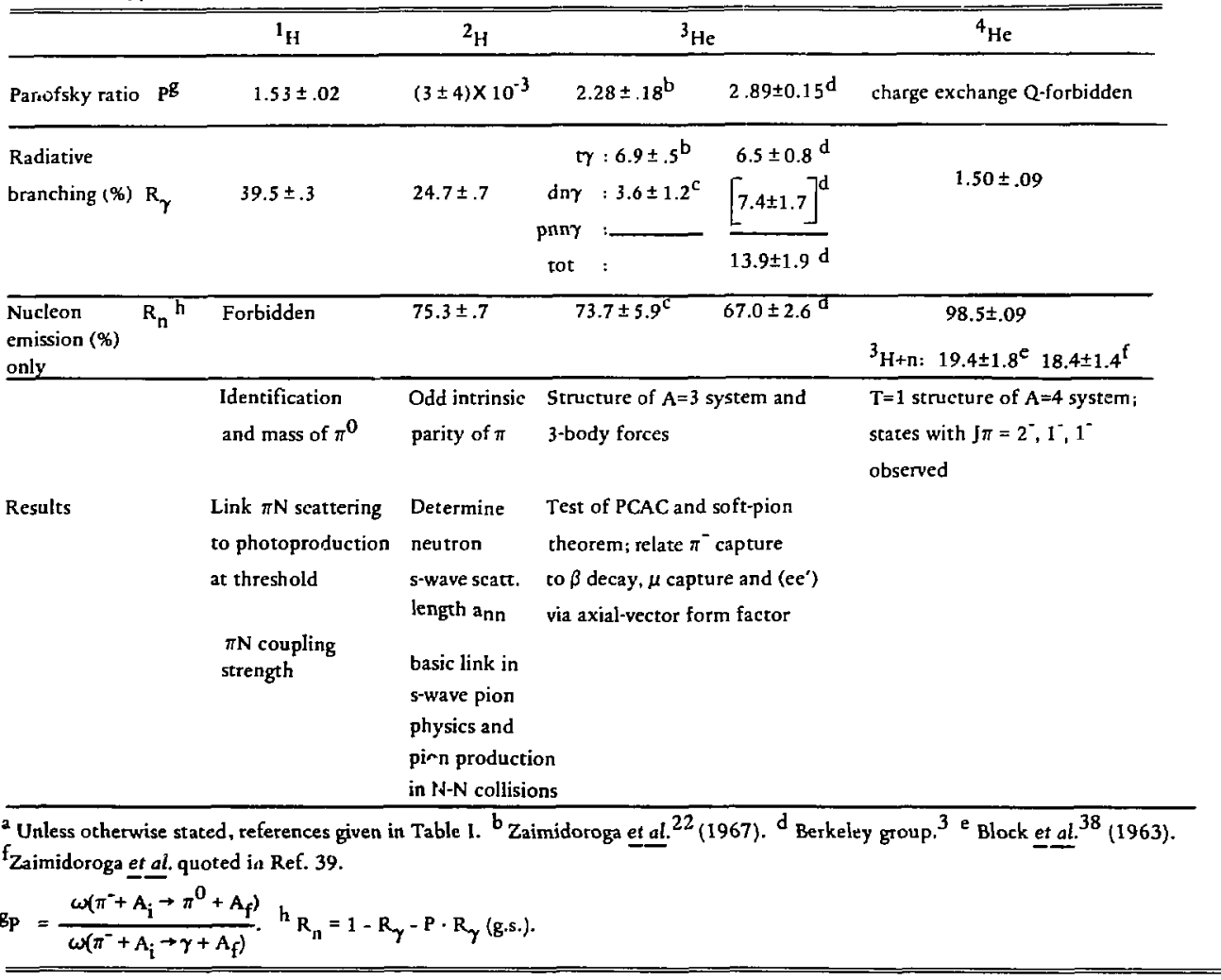

final-state $n-n$ interaction. The shape of the spectrum near its maximum can be used to determine the $n-n$ 1S-wave scattering length $a_{\text {nn }}$. Watson and Stuart 18 showed that for 1 s etate capture of the pion energy dependence of the photon spectrum near the endpoint is given by

$M^{2} \propto\left|\frac{\sin 6(p)}{p}\right|^{2} \approx \frac{1}{\left(p^{2} a^{2}+1\right)}$

$p \cot \delta=-\frac{1}{a}+\frac{1}{2} r_{0} p^{2}+0\left(r_{0}^{3} p^{4}\right)$

where $p$ is the relative neutron momentum, $a \equiv a_{n n}$ and $r_{0}$ is the effective range, assumed to beknown from pp scattering. The curve shown in Fig. $2 \mathrm{~b}$ was computed ${ }^{19}$ with $a_{n n}=16.4 \mathrm{fm}$ and $r_{0}=2.65 \mathrm{fm}$. The uncertainty in the ex- tracted ann is approximately $3 \mathrm{fm}$. The currently accepted 20 value is $a_{n n}=-16.5 \pm \sim 1.0 \mathrm{fm}$.

${ }^{3} \mathrm{He}$. The spectrum for ${ }^{3} \mathrm{He}$, displayed in Fig. $3 \mathrm{c}$, shows the presence of all four $Y$-ray channels discussed in the theoretical study of Messiah:21 (1) the $t+Y$ line at $135.8 \mathrm{MeV},(2) \mathrm{d}+\gamma$ continuum with an end-point energy of end-point energy of $129.8 \mathrm{MeV}$, (3) $p+n+n+\gamma$ continuum with an end-point of $127.7 \mathrm{MeV}$, (4) the $t+\pi^{0} ; \pi^{\circ} \rightarrow 2 \gamma$ resulting in a uniform distribution of $\gamma$ rays between 53 and $86 \mathrm{MeV}$. The relative strengths predicted by Messiah were $6.5 / 2.9 / 0.7 / 12.5$ in the order listed abcve and normalized to our measured value of the ty chanael (Table III). Our measurements do not resolve the $d y$ and pnny components, but if one uses the branching ratio for dmy determined by $Z$ aimidoroga et al. , 22 
the respective ratios are $6.5 \pm 0.8 / 3.6$ $\pm 1.2 / 3.8 \pm 2 / 18.6 \pm 2$.3. This good agree ment is remarkable considering that the calculation was performed more than 20 years ago.

The PCAC hypothesis and soft-pion theorem approach has been applied by Ericson and Figureau 23 to compute the radiative and charge exchange rates of the $\pi^{3} \mathrm{He}^{3} \mathrm{H}$ system. They obtain 2.70 for the Panofsky ratio for 1s capture which agrees well with our measurement 2.89 \pm 0.15 . Regarding $p$-state capture, for which there are no $x$-ray data, they estimate that $16 \%$ of the pions reaching the $2 p$ orbit are absorbed. Of these only $0.1 \%$ and $0.03 \%$ undergo charge exchange and radiative transitions, respectively, to the triton. Thus the Panofsky ratio should not be affected. For the absolute radiative rate from the 1 s orbit, they obtain $\lambda_{\gamma}(1 \mathrm{~s})=3.46 \times 10^{15} \mathrm{sec}$. One would like to compare this with the measured ground state branching ratio but the needed $\mathrm{p} / \mathrm{s}$ capture ratio has not been measured.

The region of the photon spectrum where the breakup channels (dny, pnny) dominate is shown in Fig. 4. The solid curve is a pole-model calculation with $\Delta=6.8 \mathrm{MeV}$ corresponding to the $\mathrm{dn \gamma}$ vertex. It is normalized to the data in the $90-$ to $115-\mathrm{MeV}$ region. This model, which neglects final-state interactions and the initial proton motion in ${ }^{3} \mathrm{He}$, is seen to give a gool overall descrption. There is a suggestion of a broad peak in the region corresponding to $10-$ to $15-\mathrm{MeV}$ excitation. This is the region where Chang et al. 24 suggest there exists a $T=1 / 2$ state on the basis of their ${ }^{2} \mathrm{H}(\mathrm{p}, \mathrm{y})^{3} \mathrm{He}$ excitation function. The statistical evidence of our spectrum, which contains a factor of nearly 4 more counts than in the fir $\mathrm{at}$ published spectrum, 25 still falls short of being totally convincing. Clearly, a careful analysis with good theoretical calculations for the two breakup channels is needed before any conclusions can be reached.

${ }^{4} \mathrm{He}$. The radiative capture rate drops by one grder of magnitude in going from $3 \mathrm{He}$ to ${ }^{4} \mathrm{He}$; indeed, the value $1.50 \pm 0.09 \%$ for ${ }^{4} \mathrm{He}$ is the lowest in Table I. The shape of the spectrum (Fig. 3d) is poorly described by the pole model, but can be entirely accounted for by assuming the excitation of three broad $T=1$ resonances: $2^{-}$at $3.4 \mathrm{MeV}$ above the ${ }^{3} \mathrm{He} \cdot \mathrm{rn}$ threshold;

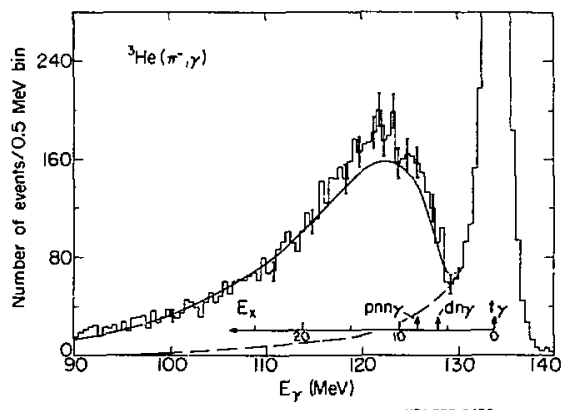

XEL 733-2432

Fig. 4. Photon spectrum for ${ }^{3} \mathrm{He}$ in region where the breakup channels pnny and dny dominate. The solid line is a pole-model calculation as discussed in the text.

$1^{-}$at $5.1 \mathrm{MeV} ; 1^{-}$at $7.4 \mathrm{MeV}$. The solid curve in Fig. $3 \mathrm{~d}$ is based on an $\mathrm{R}$-matrix calculation 4 with the simple nuclear wave functions: $\left|2^{-}\right\rangle=\left|{ }^{3} \mathrm{P}_{2}\right\rangle ;\left|1^{-}, \alpha\right\rangle$ $\left.=-0.782\left|{ }^{1} P_{1}\right\rangle+0.625^{2} \beta P_{1}\right\rangle ;\left|1^{-}, \beta\right\rangle$ $=-0.625\left|{ }^{1} \mathrm{P}_{1}\right\rangle+0.782\left|{ }^{1} \mathrm{P}_{1}\right\rangle$. The mixing between the two $1^{-}$states is not uniquely determined by the spectrum analysis. The total radiative transition rate $^{4}$ based on the above wave frunctions, $\Lambda_{\text {(ts) }}$ $=7.18 \times 10^{14} \mathrm{sec}^{-1}$, compares weil with the experimental value of $8.25 \times 10^{14} \mathrm{sec}^{-1}$ which was obtained 4 by assuming that $p-$ state capture can be neglected. A more recent calculation by Raiche and Werntz 15 which includes the $P$ state, but neglects momentum-dependent terms in the trans ition operator, yields for the radiative branching ratio $R_{y}=\left[(1.9)_{s}+(0.2)\right] \%$ $=2.1 \pm 0.7 \%$. An interesting result of the later study was to demonstrate that the neutron- $y$ correlation depends strongly on the $l$ of the pionic Bohr orbit. In the future this may provide an independent check on the capture probabilities $\omega(n)$ determined from $x$-ray data.

\section{RESULTS FOR 1P SHELL NÜCLEI}

The sensitivity of radiative $\pi$ capture to the structure of individual nuclear states is most clearly seen in the photon spectra of $1 p$-shell nuclei, $4<A \leq 16$. Examination of such spectra (Fig. 5) shows that the nuclear transition matrix elements are particularly large to certain bound and unbound states. We discuss these states separately, since the question of background subtraction and the accuracy of theoretical predictions are different. 


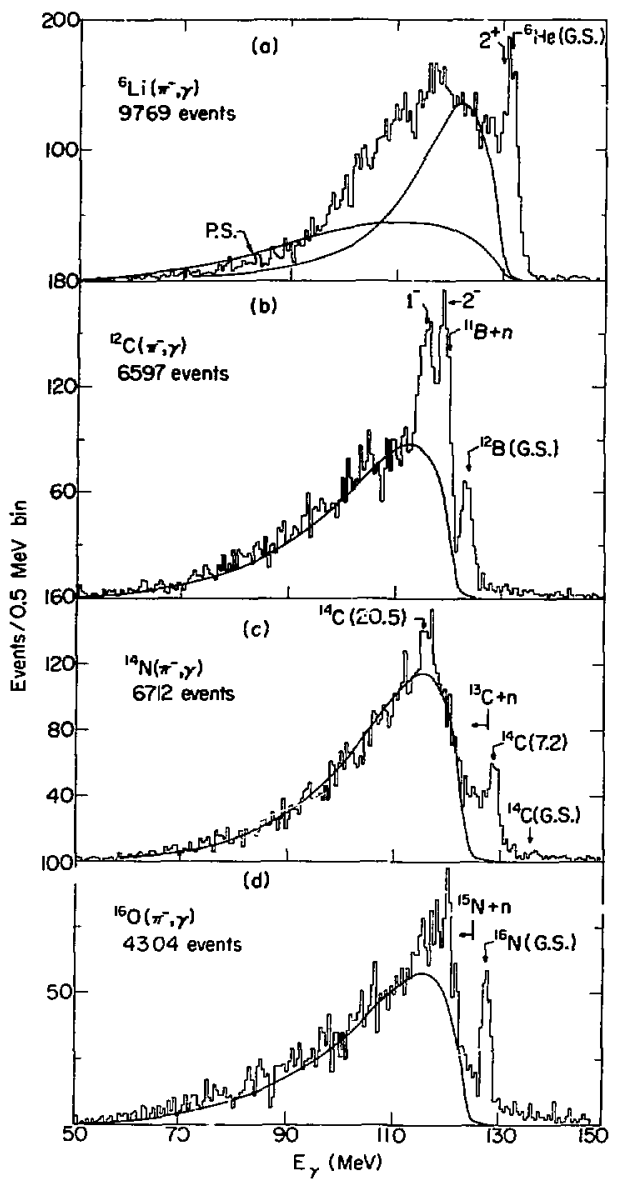

X日L $733-2435$

Fig. 5. Photon spectra for 1p-shell nuclei. Solid curves are pcle-model calculations. The phase space curve (P.S.) in (a) is seen not to describe th usiasumen well.

\section{GIANT RESONANCES}

As was mentioned previously, the motivation for performing high-resolution radiative $\pi$-capture studies was based largely on interest in observing giant resonances, particular the giant dipole (GD) states. The states to be observed are the $\mathrm{I}_{\mathrm{z}}=+1$ members of $\mathrm{T}=1 \mathrm{iso-}$ baric triads whose $T_{z}=0$ members are the giant resonances well known from photonuclear and inelastic electron-scattering studies. Strong excitation of such states in $\pi$ capture was predicted by Delorme and Ericson' (1966), Anderson and Eisenberg ${ }^{2}(1966)$, and Überall 2 (1966). Bistirlich et al. 4 first observed

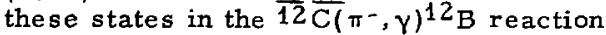
and their results are shown in Fig. 1. The spectrum shows three peaks at photon energies of 124.7, 120.3, and 116 . 9 $\mathrm{MeV}$ which were identified with transitions to $1^{+}, 2^{-}$, and $1^{-}$states, respectively, in $12 \mathrm{~B}$. The analogs in $12 \mathrm{C}$ are at 15.1 , 19.9, and $23.7 \mathrm{MeV}$. The solid curve in Fig. 1c shows the fit to the data assuming three Breit-Wizner forms. The data in this figure are what remain after subtraction of a contribution due to in-flight capture (transitions before $\pi^{-}$comes to rest) and the quasi-free component as given by the pole model. 7 The dashed curve shows the spectrum predicted by Kelly and itberall, 27 using the $12 \mathrm{C}$ wave functirns of Kamimura et al. 27 These wave functions, tailored to describe the splitting of the GD state observed in the ${ }^{11} \mathrm{~B}(\mathrm{p}, \mathrm{y})^{\mathrm{P}} \mathrm{C}$ reaction, couple the $2^{+}$state at $4.43 \mathrm{MeV}$ in $12 \mathrm{C}$ with the GD os cillation. When used to compute the $\pi$ capture rates, they are seen to result in a qualitative description of the data.

Skupsky ${ }^{13}$ has improved the theoretical analysis of the ${ }^{12} \mathrm{C}$ data. He included the momentum-dependent terms in the transition operator of Eq. (5) and used nuclear wave functions for the giant resonances which coupled an s-d shell nucleon to lowenergy states of the Cohen-Kurath model for $11_{C}$. His calculations show that three states dominate photoexcitation, $\mu$ capture, and $\pi$ capture: two 1- levels computed to be at 22.4 and $25.9 \mathrm{MeV}$ in ${ }^{12} \mathrm{C}$, and one $2^{-}$level at $20.6 \mathrm{MeV}$. The two $1^{-}$ states are largely responsible for the oiserved splitting of the GD strength in photoabsorption. The $2^{-}$state, not seen in photoabsorption, dominates $\mu$ capture where its strength results entirely from the axial vector interaction. In $\pi$ capture, all three levels are strongly populated and are identified with the peaks in the photon spectrum at 120.3 and 116.9 $\mathrm{MeV}$. The predicted radiative branching ratios (Table IV) to these states are $R_{Y}\left(1^{-}\right)=0.19 \%$ and $R_{Y^{\prime}}(2)=0.28 \%$. The value of Bistirlich et $l_{1}^{4}$ are $R_{y}\left(1^{-}\right)$ $=0.159 \pm 0.016 \%$ and $R\left(2^{-}\right)=0.185 \pm 0.019 \%$. A likely explanation for the discrepancy in $\mathrm{R}_{\mathrm{y}}\left(\mathrm{2}^{-}\right)$is that in the experimental de . termination the pole-model subtraction is too large. If no pole-model subtraction is 
Table IV. Comparison of experimental and theoretical radiarive $\pi$-capture branching ratios in Ip-shell nuelei.

\begin{tabular}{|c|c|c|c|c|c|c|c|c|}
\hline \multirow{2}{*}{$\begin{array}{l}\text { Nuclei } \\
\left|T_{z}=0\right\rangle \rightarrow\left|T_{z}=1\right\rangle\end{array}$} & \multirow{2}{*}{$\mathrm{j}_{\mathrm{i}}^{\pi}$} & \multirow[b]{2}{*}{$\mathrm{J}_{\mathrm{f}}^{\pi}$} & \multicolumn{2}{|c|}{ Experiment ${ }^{a}$} & \multirow[b]{2}{*}{$\begin{array}{l}\mathbf{R}_{\boldsymbol{\gamma}} \\
(\%)\end{array}$} & \multicolumn{2}{|l|}{ Shell model } & \multirow{2}{*}{ 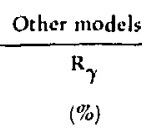 } \\
\hline & & & $\begin{array}{c}\mathrm{E}_{\mathrm{x}}\left(\mathrm{T}_{\mathrm{z}}=1\right) \\
(\mathrm{McV})\end{array}$ & $\begin{array}{c}\mathrm{E}_{\mathrm{x}}\left(\mathrm{I}_{\mathrm{z}}=0\right) \\
(\mathrm{MeV})\end{array}$ & & $\begin{array}{l}\mathrm{R}_{\gamma} \\
(\%)\end{array}$ & $\begin{array}{l}\text { Valence } \\
\text { Orbitals }\end{array}$ & \\
\hline \multirow[t]{2}{*}{${ }^{6} \mathrm{Li} \rightarrow{ }^{6} \mathrm{Hc}$} & $1^{+}$ & $0^{+}$ & 0.0 & 3.56 & $.306 \pm .035$ & $.36^{\mathrm{b}}$ & $1 \mathrm{p}$ & $.41 \pm .11^{2}$ \\
\hline & & $\hat{i}^{+}$ & 1.8 & 5.36 & $.148 \pm .025$ & $.08^{\mathrm{b}}$ & Ip & \\
\hline \multirow[t]{3}{*}{${ }^{12} \mathrm{C} \rightarrow{ }^{12} \mathrm{~B}$} & $0^{+}$ & $\left(1^{+}, 2^{+}, 2^{-}\right)$ & .35 & 15.45 & $.091 \pm .009$ & $.07^{d}$ & $1 p, 2 s, 1 d$ & $.105 \pm .035^{\circ}$ \\
\hline & & 2 & 4.75 & 19.85 & $.385 \pm .019(.30)^{g}$ & $.28^{\mathrm{e}}$ & $1 p, 2 s, 1 d$ & \\
\hline & & $1^{-}$ & 8.10 & 23.20 & $.159 \pm .016(.42)^{g}$ & $.19^{f}$ & $1 \mathrm{p}, 2 \mathrm{~s}, 1 \mathrm{~d}$ & \\
\hline \multirow[t]{4}{*}{${ }^{14} \mathrm{~N} \rightarrow{ }^{14} \mathrm{C}$} & $1^{+}$ & $0^{+}$ & 0.0 & 2.31 & $<.008$ & $.008^{h}$ & $1 p$ & \\
\hline & & $2^{+}$ & 7.01 & $9.17\}$ & $001+02$ & $.12^{h}$ & tp & \\
\hline & & $2^{-}$ & 7.34 & $9.51\}$ & $.094 \pm .024$ & $.009^{h}$ & $1 \mathrm{p}, 2 \mathrm{~s}, 1 \mathrm{~d}$ & \\
\hline & & Giant res. & 20.5 & 22.8 & & & & \\
\hline \multirow[t]{3}{*}{$16_{0} \rightarrow 16_{N}$} & $0^{+}$ & $(2,0,3,1)$ & 0.0 & 13.0 & $.15 \pm .03$ & $.168^{k}$ & $\left\{\begin{array}{l}1 \mathrm{p}, 2 \mathrm{~s}, 1 \mathrm{~d} \\
2 \mathrm{p}, 1 \mathrm{f}\end{array}\right.$ & \\
\hline & & $2^{\circ}$ & 7.70 & 20.7 & $.22 \pm .05(.58)^{g}$ & & & $.43^{\mathrm{i}}$ \\
\hline & & $\mathrm{GD}+\mathrm{GQ}$ & $5.6-15.6$ & $18.6-28.6$ & $.25 \pm .06(.97)^{g}$ & & & $1.65^{\mathrm{j}}$ \\
\hline
\end{tabular}

${ }^{a}$ Berkeley group. ${ }^{3}$ b Vergados and Baer ${ }^{14}$ (1972). ${ }^{c}$ Maguire and Werntz ${ }^{9}$ (1972). ${ }^{d}$ Skupsky ${ }^{13}$; includes unly $2^{-}$state at Ex $=5.1 \mathrm{MeV}$.

Skupsky ${ }^{13} ; 1^{-}$states at $\mathrm{Ex}=7.0,7.5,10.5 \mathrm{MeV}, \mathrm{B}$ Assume pole-model contribution $=0$. ${ }^{\mathrm{h}} \mathrm{Vergados}^{31}(1972)$.

$i_{\text {Murphy et al. }}^{28}$ (1967); transition to $2^{-}$state predicted at $17.5 \mathrm{MeV}$ in ${ }^{16} \mathrm{O}$.

$\mathrm{j}_{\text {Murphy et al. }}{ }^{28}(1967)$; sum of contributions to GD states $\left(0^{-}, 1^{-}, 2^{-}\right)$and $\mathrm{GQ}$ states $\left(1^{+}, 2^{+}, 3^{+}\right)$between 17.5 and $29.1 \mathrm{MeV}$ in $16 \mathrm{O}$.

$k_{\text {Werntz }} 30$ (1971). 
made, one obtains $0.30 \%$-much closer to the theoretical value.

In ${ }^{16} \mathrm{O}$, the giant quadrupole resonances $(L=2, S=1, T=1)$ were predicted to be important by Murphy et al. 28 Using a generalized Goldhaber-Teller model, they showed that for 1s capture the photon spectra of $\pi$ capture are dominated by giant dipole states, for $2 p$ capture by giant quadrupole (GQ) states $\left(1^{+}, 2^{+}, 3^{+}\right)$. Since more than $90 \%$ of the pions are captured from $p$ orbitals, it was hoped that evidence for these nottoo-well-established collective modes could be found. Unfortunately, the spectrum in the region of the GQ states, $E_{y}=111.5$ to $121.5 \mathrm{MeV}$, shows no easily identified resonances. Bistirlich et al. 4 get a branching ratio of $\sim 0.25 \%$ for the strength to this region after they subtract the non-resonant component by the pole model. Murphy et al. get $1.65 \%$ for the total strength to $\overline{G D}$ and $G Q$ states in this region. If one assumes in the determination of the experimental number that the quasi-free component is zero in this region, the branching ratio is $0.97 \%$, which is closer to the theoretisal estimate. Nevertheless, one must say that the evidence is inconclusive. It would be useful to have a calculation for GQ states, using the full Hamiltonian and better nuclear wave functions.

The preliminary results of ${ }^{14} \mathrm{~N}$ (Fig. 5c) show a resonance-like peak at 20.5 $\mathrm{MeV}$ in ${ }^{14} \mathrm{C}\left(22.8 \mathrm{MeV}\right.$ in $\left.{ }^{14} \mathrm{~N}\right)$. It will be interesting to see if the prediction by Murphy et al. that $G Q$ excitations dominate when the pions get captured predominantly from $l=1$ orbits can be verified. The subtraction of the quasi-free background may be clearer in $14 \mathrm{~N}$ since, unlike ${ }^{12} \mathrm{C}$ and 160 , this component seems to extend on both sides of the resonance. One sees that a larger value of the excitation energy of the recoil nucleus $E_{x}=\Delta-\Delta$ (min)must be used in the pole model. For the curve shown in Fig. $5 \mathrm{c}$, $E_{X X}=3.9 \mathrm{MeV}$. In the $12 \mathrm{C}$ and 160 analysis, $E_{x}=0$ and $1.6 \mathrm{MeV}$ respectively were employed. The fit to the $14 \mathrm{~N}$ data given by the pole model is excellent (the full analysis of this spectrum is still in progress). The larger $\Delta$ needed in ${ }^{14} \mathrm{~N}$ suggests that the ${ }^{2} \mathrm{C}$ and $16_{0} \mathrm{data}$ may require a large $\Delta$. As discussed above, this would improve the agreement with theory on giant-resonance excitation. identified in the mass -6 system. The suggestion of a peak occurs at $E_{y}=119 \mathrm{MeV}$ (Fig. 5a). This is at $15.6 \mathrm{MeV}$ relative to the $6 \mathrm{He}$ ground state and $3.3 \mathrm{MeV}$, above the threshold for breakup of $6^{6} \mathrm{He}$ into two tritons. Possibly, the observed structure is related to this channel.

There are suggestions of other resonances in the spectrum, but the statistical evidence is weak. One can see that the pole model does not describe the data as well as for the other ip shell nuclei. The spectrum expected for quasi-free capture on a deuteron in ${ }^{6} \mathrm{Li}$ is recently been calculated 29 (not shown). The photon spectrum for this capture mode makes relatively large contributions to the 90 to $120-\mathrm{MeV}$ region, the region where the pole model for quasi-free capture on a proton deviates most from the data. A more complete study 29 of quasi-free capture on heavier clusters such as deuteron, and $\alpha$-particles is in progress.

\section{BOUND STATES}

Since the nuclear structure of bound states is much better understood theoretically, and the experimental branching ratios are nearly free from uncertainties in background substraction, one hopes for a precise evaluation of radiative $\pi$-capture theory. This is particularly true for the odd-odd nuclei ${ }^{6} \mathrm{Li}_{\mathrm{i}}$ and $14_{\mathrm{N}}$ where transitions to individual states can be resolved.

In the ${ }^{12} \mathrm{C}$ study, the ground state group (Fig. 1) is in the region of the $1^{+}$, $2^{+}$, and $2^{-}$states in ${ }^{12} \mathrm{~B}$ at $0.0,0.95$, and $1.67 \mathrm{MeV}$, respectively. The measured excitation energy, $0.35 \pm 0.05 \mathrm{MeV}$, shows that the 1+ transition dominates, but that there is a non-negligible contribution from one or both of the other two states. Skupsky ${ }^{13}$ obtains (Table IV) for the branching ratios $1^{+}\left|2^{+}\right| 2^{-} \mid$sum the values, in $\%, 0.045|0.021|<0.01 \mid 0.066$. Maguire and Werntz 9 obtain $0.054|0.036|$ $0.014 \mid 0.105$, respectively. The agreement with the measurement $\mathrm{R}_{\mathrm{f}}$ (sum) $=0.091 \pm 0.009$ is seen to be quite good, but one would like better $x-r$ ay data on the $2 p$ state before making final judgements.

For ${ }^{16} \mathrm{O}$ there have not been many theoretical calculations for the bound state transitions. The observed ground state group fully spans the region of the $2^{-}, 0^{-}, 3^{-}$, and $1^{-}$states in $16 \mathrm{~N}$ at 0.0 , $0.12,0.30$, and $0.40 \mathrm{MeV}$, respectively. The measured branching for the sum of 
states is $0.11 \pm 0.02 \%$. A theoretijgal value of $0.168 \%$ was given by Werntz, ${ }^{30}$ using the wave functions of Szydlik and Philpot. 30

The ${ }^{14} \mathrm{~N}$ spectrum provides a unique possibility for studying $\pi$ capture: the combination of high neutron separation energy ${ }^{14 \mathrm{C}}(8.2 \mathrm{MeV})$ and high excitation energy of the first excited state in 12C $\left(1^{-}, 6.1 \mathrm{MeV}\right)$ permits the ground state to be completely resolved. One sees in Fig. 5c that the transition is extremely weak. The $\beta$ transition between the two states exhibits the well-known anomaly, i.e. , $\log \mathrm{ft}=9$ rather than 3 as expected for an allowed Gamow-Teller transition. The radiative $\pi$-capture transition from the 1s-state also predominantly a GamowTeller transition (but at a higher momentum transfer), might therefore be expected to be small. The radiative $p$-state capture is apparently also small, judging from the small upper limit $R_{Y}<8 \times 10^{-5}$ set by the experiment. Vergados 31 analyzed this transition with shell-model wave functions and obtained $R_{\gamma}\left(0^{+}\right)=7.7 \times 10^{-5}$. The other bound state peak seen in the spectrum is at $7.2 \mathrm{MeV}$ excitation in ${ }^{14} \mathrm{C}$. In this region the re is $2^{+}$and $2^{-}$state at 7.01 and $7.34 \mathrm{MeV}$, respectively.

Vergados 31 predicts (Table IV) that the $2^{+}$ state completely dominates this region. The combined strength, $0.13 \%$, compares well with the measured value $0.094 \pm 0.024 \%$. In addition, Vergados finds that all other states below $9.5 \mathrm{MeV}$ in ${ }^{14} \mathrm{C}$ make small contributions to the spectrum.

\section{PCAC AND SOFT -PION APPROACH} COMPARED WITH IA CALCULATIONS AND DATA IN 6 Li.

The transition $\pi^{-}+6 \mathrm{Li}\left(1^{+}\right) \rightarrow \gamma^{+}{ }^{6} \mathrm{He}$ $\left(0^{+}\right.$, g. s. ) provides an important test of the PCAC hypothesis and soft-pion theorem approach (EP) for complex nuclei. The necessary input for the prediction of the $1 \mathrm{~s}$ radiative capture rate $\lambda_{Y}(1 \mathrm{~s})$ is available: the $\beta$-decay and $\mu$-capture rates are measured, the $0^{+}$analog state in ${ }^{6} \mathrm{Li}$ at $3.562 \mathrm{MeV}$ has a well measured Y-decay width, and the electron scattering to this state has been measured by several groups. Thus a reasonably good determination of the axial vector form factor is possible. The experimental $\pi$ capture branching ratio has been determined by Deutsch et al. 32 and the Berkeley group. 33

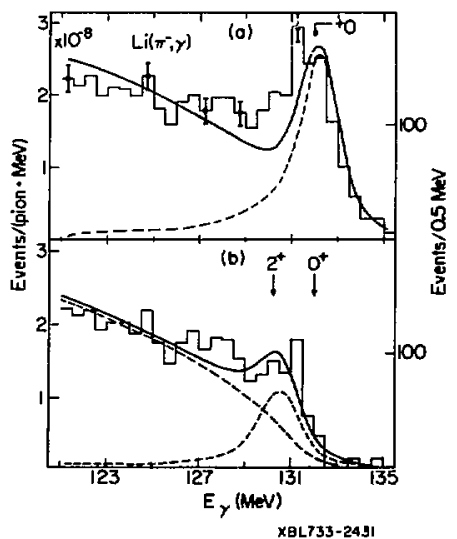

Fig. 6. High-energy end of ${ }^{6} \mathrm{Li} \mathrm{spec-}$ trum. (a) Single-line fit; dashed curve shows instrumental line shape. (b) Data after subtraction of ground state line; dashed curves: assumed shape of continuum and line at $\mathbf{E}_{\mathbf{x}}=1.80 \mathrm{MeV}$ corre sponding to excitation of $2^{+}$state.

The photon spectrum in the region of the $0^{+}$state is shown in Fig. 6. The comparison of the instrumental line shape with the peak in the $6_{\mathrm{Li}}$ spectrum indicates that the latter is too broad for a single transition. A careful analysis 33 indicates that one must assume population of the $2^{+}$ state at $1.8 \mathrm{MeV}$ as shown in Fig. 6b. The uncertainty that the presence of the $2^{+}$ state introduces on the extraction of the gruund state is small, since we can normalize a single line to the $6_{\text {Li }}$ spectrum above $132 \mathrm{MeV}$ where the contribution from other channels is small.

Four groups of authors have used the PCAC hypothesis and the soft-pion limit to compute $\lambda_{Y}(1 \mathrm{~s})$. These are given in Table $\mathrm{V}$ together with the results of Maguire and Werntz, 9 who use the IA Hamiltonian but relate the nuclear matrix elements to other measured rates, and the shell model calculations of Roig and Pascual 11 and Vergados and Baer. 14

We note these points:

(1) $\Lambda_{\gamma}$ (1s): The EP values agree in general with IA. values; however, the average EP value (excluding the 1968 estimiate, which did not include some corxections) $\lambda_{y}(1 \mathrm{~s})=2.03 \times 10^{15} \mathrm{sec}^{-1}$ is higher than the average IA value $1.46 \times 10^{15} \mathrm{sec}^{-1}$. The EP value of Delorme 34 (2.3 \pm 0.5$)$ $\times 10^{15} \mathrm{sec}^{-1}$ is the highest. 
Table V. Radiative transition rates and branching ratios for $\pi_{\text {stop }}^{-}+{ }^{6} \mathrm{Li}\left(1^{+}\right)-{ }^{6} \mathrm{He}\left(0^{+}\right)+\gamma$

\begin{tabular}{|c|c|c|c|c|c|c|}
\hline \multirow[t]{3}{*}{ Method } & \multicolumn{2}{|c|}{ Transition rates } & \multicolumn{3}{|c|}{ Branehing ratios" } & \multirow[t]{3}{*}{ Reference } \\
\hline & $\lambda_{\gamma}(1 s)$ & $\lambda_{\gamma}(2 p)$ & $\mathbf{R}_{\mathbf{s}}$ & $\mathbf{H}_{\mathbf{p}}$ & $R_{\gamma}(s+p)$ & \\
\hline & $10^{15} \sec ^{-1}$ & $10^{10} \sec ^{-1}$ & (x) & (x) & (x) & \\
\hline PCAC & 1.65 & & $.289 \pm .118$ & & & Griffiths, Kim (1968) ${ }^{34}$ \\
\hline$\cdot$ & $1.86 \pm .18$ & & $.326 \pm .134$ & & & Pascual. Fujii (1970) ${ }^{34}$ \\
\hline \multirow[t]{2}{*}{ soft pions } & $\begin{array}{r}1.9+.4 \\
. .2\end{array}$ & & $.333 \pm .136$ & & & Fulches, Fisenberk $(1970)^{34}$ \\
\hline & $2.3 \pm .5$ & & $.404 \pm .: 66$ & $.312 \pm .047$ & .7164 .171 & Delorme $(1970)^{34}$ \\
\hline IA & $1.51 \pm .15$ & $5.26 \pm .06$ & $.265 \pm .109$ & $.1384 .0+3$ & $.403 \pm .117$ & McGuirt. Werniz $(1973)^{9}$ \\
\hline $\mathbf{t \wedge}$ & $1.46 \pm .22$ & $4.12 \pm .62$ & $.256 \pm .155$ & $.10 \$ \pm .033$ & $.364 \pm .111$ & Pascusl, Roiz $\{1972)^{n !}: \quad=1.94 \mathrm{fm}$ \\
\hline$\cdot$ & 2.00 & 4.32 & $.365 \pm .150$ & $.113 \pm .035$ & .4795 .154 & Vergadus. Aacr (1972) ${ }^{14}$; $-1.66 \mathrm{fm}$ \\
\hline Shell model & 1.40 & 4.44 & $.244 \pm .101$ & .1172 .036 & $.565 \pm .107$ & Verexdon, Haer $(1972)^{14}:$ a $1.94 \mathrm{fm}$ \\
\hline Average $(1 \mathrm{~A})^{b}$ & 1.46 & +.61 & $.256 \pm .105$ & .1212 .037 & .5725 .111 & \\
\hline Experiment & & & & & $\begin{array}{l}1.0 \leq 1.1 \\
.306 \leq .035\end{array}$ & 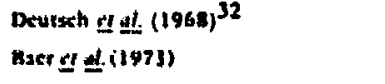 \\
\hline
\end{tabular}

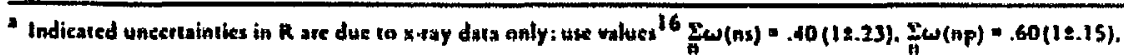

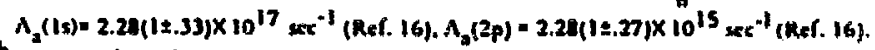

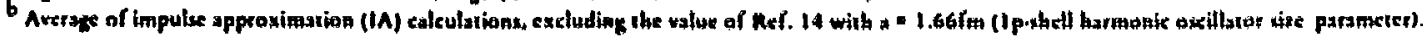


(2) $\lambda_{1}\left(z_{p}\right)$ : Since the oft-pion limit does nol apply to $2_{p}$ e apture, only $U$ values are avallable. All etimates in Table $Y$ exeept that of Delorme: are close to the average $y_{y}(z)=4.61 \% 10^{10}$ see ${ }^{-1}$ (excludingt that of Ret. 14 ).

(3) R: The average in value $0.378 \pm 0.111$ to agrew with our value $0.306 \pm 0.035 \%$ but disagrees with the $1.0 \neq 0.1 \%$ of Deutsth al al. 32

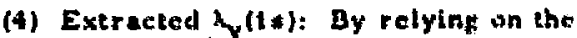
IA extimate for A(2p). one ean exe ret tys).

$$
\begin{aligned}
y_{Y}(1 *) & =\frac{R_{x}(\operatorname{espl})-R_{p}(1 A)}{w_{s}} \times R_{A}(1:) \\
& =(1.05 \pm 0.52) \times 10^{15} \sec ^{-1} .
\end{aligned}
$$

This is in agreement with in outimate: but some what lower than the EP value:

In concluston, we ean *ay that at the present level of comparieon between cheory and sxperiment. no major discropancles with either the EP or $\mathrm{A}$ eal. culations are *een. Clearly more precise $x$-ray data are dewirable. As these become available it should be possible to extract a precise value of $h_{y}(i s)$ which would test with greater accurecy the PCAC hypothests and coft-pion approseh.

\section{MEDIUM-MASS AND MEAVY NUCLEt}

All hiph-resolution data now available on nuelei with $A>16$ are dieplayed in Fig. 7. The very preliminary data on $209 \mathrm{Bi}$ is dirse presented here. The tolal radiative capture rates (Table I) aret nearly the ame for these three nuelei. although the eapture orbitgls are differrnt. The Bohe radit $\left(\mathrm{ra}_{\mathrm{B}}=200 \mathrm{n}^{2} / 2 \mathrm{fm}\right)$ and nuclear radll $(\mathrm{r}=1.2 \mathrm{~A} 1 / 3 \mathrm{fm})$ in order $24 \mathrm{Mg}(2 \mathrm{p}$. $40 \mathrm{Ca}(3 \mathrm{~d}), 209 \mathrm{BI}(5 \mathrm{~d})$ are 67.90 , $60 \mathrm{fm}$ and $3.5,4.1,7.1 \mathrm{fm}$, respetively. One sees that the everase distances between the pion and the proton when the interaction occurs do not vary preatly in the se nuelei.

The photon spectra do not elearly show excitation of individual nuclear levels. How ever, one can ece for $24 \mathrm{Mg}$ and $40 \mathrm{Ca}$ that there is considerable strength to the bound states. One cannot help feeling that if the spectra were recorded with a

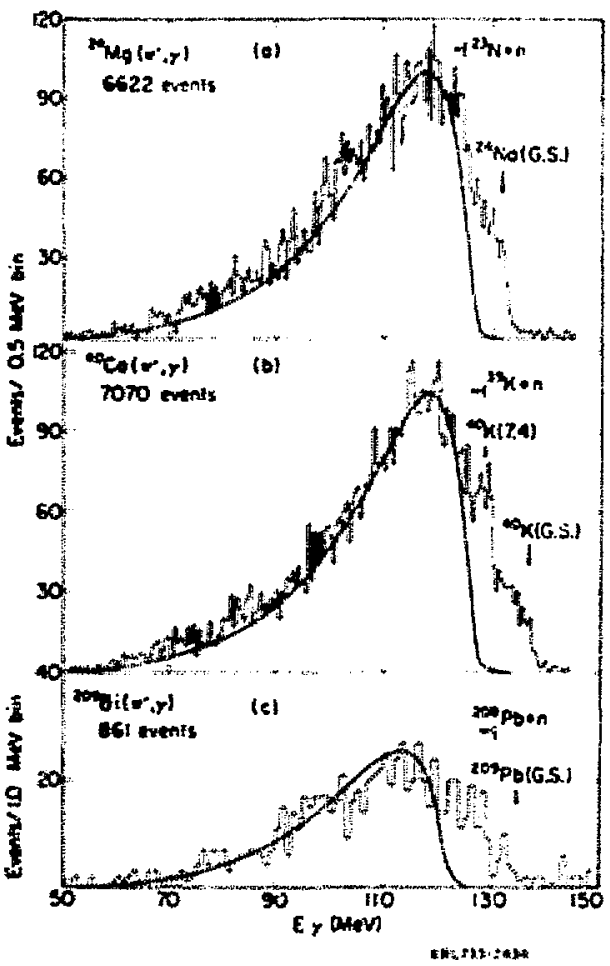

Ftz. 7. Photon pectra for medium mast and heavy nuclei. The eurves are pole-model exleutations. Evidence for excitation of the bound states of $24 \mathrm{Na}$ and tok ean be seen clearly.

rowolution closer to that achieved in low. cnerey nuelear physics, say $300 \mathrm{keV}$, a new and rich spectroscopy of nuclear states would emergo. The fact that the basfe transition operator is much better understood than, tor example, the much used (p.p') reaction al 50 AinV, would make it a very powerful tool tor nuelear structure investigations. With higher intensity plon beams soon to become avail. able, the propects seem favorable for obtaining 300-xeV resolution with a pair spectrometer making use of a thinnet converter loil.

The authors wish to thank Drs. John Vergados. Gary Nixon, and Carl Wernte for exmmunicating unpubltshed results and for nume rous helplul discuseions. 


\section{REFERENCES}

${ }^{1} H$. Davies, H. Muirhead, and T. N. Woulds, Nucl. Phys. 78, 673 (1966). ${ }^{2}$ J. Delorme and T. E. O. Ericson, Phys. Letters 21, 98 (1966); D. S. Anderson and J. $M$ Eisenberg. Phya. Letters 22, 164 (1966): H. Uberall, Nuovo Cimento Suppl. 1, 781 (1966). 3 Berkeley roup (past and present): $J$ A. Bistirlich, N. de Botton, S. C. Cooper. J. A. Helland, A. S. L. Parsons, Paul Skarek. P. Truol and the authors.

4. A. Bistirlich et al. , Phys. Rev. Letters 25, 689 (1 द7L); ibid. 25, 950 (1972): Phys. Rev. C 5, 1867(1972). 5 geviewed by M. Ericson and M. Rho, Phys. Reports 5C. 59 (1972).

${ }^{6}$ C. W. Kim and H. Primakoff, Phys. Rev. 139, B1447 (1965).

7L. G. Dakhno and YU. O. Prokoshkin, Sov. J. Nucl. Phy*. 7. 351 (1968); I. S.

Shapiro, Selected Topics in Nuclear Theory (MEX), Vienna 1963), p. 85 ff.

M. Kawaguchi ce al. . Prog. Theor. Phys. Suppl. (Kyoto), Extra No. 28.

${ }^{9}$ W. Maguire and C. Werntz, Nucl. Phys., in press.

${ }^{10} \mathrm{G}$. Nixon, private communication (1973);

D. Schwela, private communication to

P. Truot (1973).

11F. Roig and P. Pascual, preprint,

G. I. F. T. (Spain) (April 1972).

${ }^{12}$ F. A. Berends. A. Donnachi, and D. Weaver. Nucl. Phys. B4, 1 (1967).

${ }^{13}$ S. Skupsky, Phys. Letier 3́g. 271

(1971): Nucl. Phy*. A178, 289(1971).

i4 J. D Vergados and H. W. Baer, Phys.

Letters B41, 560 (1972); J. D. Yezgados. private communication.

15 A. Raiche and C. Werntz, Phys. Rev. C 1. 2003 (1971), and references therein. 16. W. Sapp et al. , Phys. Rev. C 5. 690 (1972); R. J. Harric et al. , Phys. Rev. Letters 20, 505 (1969).

17M. Leon, Phys. Letters 37B, 87 (1971).

$18_{K}$. M. Watson and R. N. Stuart, Phye.

Rev. 82, 738 (1951).

19 J. W. Ryan, Phys. Rev. 130, 1554 (1963).
${ }^{20}$ H. Brũckman, (rapporteur), Int. Conf. on Few Part. Prob. Nucl. Int. , Los Angeles, 1972.

21 A. M. L. Messiah, Phys. Rev. 87, 639 (1952).

22 o. A. Zaimidoroga et al. , Soviet Phys.

(JETP) 21, 848 (1965), ibid. 24, 1111 (1967).

${ }^{23}$ M. Ericson and A. Figureau, Nucl. Phys. B3, 609 (1967).

${ }^{24}$ C. C. Chang, E. M. Diener, and E. Ventura, Phys. Rev. Letters 29, 307 (1972).

${ }^{25}$ H. W. Baer et al. Int. Conf. Few

Part. Prob. Nucl. Int. , Los Angeles, 1972.

27 F. J. Kelly and H. Überall, Nucl. Phys. A118, 302(1968); M. Kamimura et al., ibid. A95, 129 (1967).

28. D. Murphy et al. , Phys. Rev.

Letter: 19, 714 (1967).

${ }^{29}$ G. Nixon et al., Bull. Am. Phys. Soc. 1973 (Washington)

${ }^{30} \mathrm{C}$. Werntz, invited paper, Am. Phys. Soc. meeting, Tuscon, 1971 ; nuclear wave functions from P. Szydlik and I. Philpot, Phys. Rev. 153, 1039 (1967).

31 J. D. Vergados, contribution to this conference.

32 J. Deutsch et al. , Phys. Letters 26B. 315 (1968).

${ }^{33} \mathrm{H}$. W. Baer et al. to be submitted to Phys. Rev.

${ }^{34}$ J. Delorme, Nucl. Phys. B13, 573

(1970); L. P. Fulcher and J. $M$.

Eisenberg, ibid. B18, 271 (1970);

P. Pascual and A. Fujii, Nuovo Cimento 65A, 411 (1970); Exxatum 67A, 135 (1970).

${ }^{35}$ G. Backenstos s, Ann. Rev. Nucl.

Science 20, 467 (1970).

${ }^{36} \mathrm{~W}$. Chinowsky and J. Steinberger, Phys.

Rev. 100, 1476 (1955).

${ }^{37}$ y. I. Petrukhin and Yu. D. Prokoshkin, Nucl. Phys. 54, 41.4 (1964).

${ }^{38}$ M. M. Block et al. , Phys. Rev.

Letters 11, 301 (1963).

${ }^{39}$ T. I. Kopaleishvili, Particles and Nuclei 2 (part 2), 87 (1973).

${ }^{40}$ G. F. Chew et al. Phys. Rev. 106, 1345 (1957). 